\title{
Quality Grading of Locally Grown Snap Beans for the Fresh Market ${ }^{1}$
}

\author{
A. J. Rodriguez, R. Guadalupe, and J. R. Cruz ${ }^{2}$
}

\begin{abstract}
Snap bean samples from the fall, winter and spring plantings were of higher quality than those collected from similar varieties planted in the summer. The Astro and Tendergreen from the summer plantings were the best producers of graded beans, although their yields were only 63.8 and $63.9 \%$, respectively. CV Harvester from the fall and spring plantings picked at 45 and at 52 days after planting produced the most No. 1 grade beans among the six varieties of the study.
\end{abstract}

\section{INTRODUCTION}

A major program is now in progress in Puerto Rico for the commercial production of vegetables. This program has been undertaken because of the demand of the consumers for high quality fresh produce. The local vegetable market amounts to approximately $\$ 70$ million yearly, a large proportion of which is imported in processed form. About $\$ 2$ million accounts for the wholesale value of snap beans alone. Most of the imported vegetables are quality graded according to USDA grading standards.

Because of the local conditions of climate and soil that are very well suited for the development of an efficient vegetable industry, a substantial part of the market of vegetables could be supplied from local sources. But for successful competition with imported produce, realization of vegetable production requires studies on grading to determine the extent to which the quality of the locally grown vegetables conforms with USDA grading standards.

We present herein information collected in the study of quality grading of six varieties of snap beans planted during fall 1974, winter 1974-75, spring 1975, and summer 1975.

\section{MATERIALS AND METHODS}

Six varieties of snap beans-Contender, Astro, Wade, Tendergreen, Harvester and Olympia-were planted at the Isabela Substation during fall and winter 1974 and during spring and summer 1975.

The plantings were established in adjacent plots $9 \mathrm{ft} \times 100 \mathrm{ft}$ on a Coto clay (Oxisol) soil to which fertilizer 10-10-8 was applied at planting.

' Manuscript submitted to Editorial Board July 20, 1978.

${ }^{2}$ Chemical Engineer, Assistant Horticulturist and Assistant Food Technologist, respectively, Food Technology Laboratory, Agricultural Experiment Station, Mayagüez Campus, University of Puerto Rico, Río Piedras, P. R. 
Overhead irrigation was also applied regularly until flowering. Dacthal ${ }^{3}$, a pre-emergent herbicide, was used immediately after planting, followed by weekly sprayings of Diazinon ${ }^{3}$ and Dithane $\mathrm{M}-45^{3}$ to fight insects and fungi.

Samples were collected as follows: beans planted in September 1974 were harvested in November 1974. The December 1974 plantings were harvested in February 1975. Beans planted in March 1975 were harvested in June; those planted in June were collected in August. Two hand pickings or samplings were made from each plot of the fall, winter and spring seasons: one 45 days after planting, and a second about 7 days later. Sampling of the summer plots started 47 days after planting and continued at 2-day intervals thereafter until plants were 61 days old.

Pods were graded for quality and also submitted to a panel of tasters for sensory evaluation. For the quality evaluation, samples of 560 grams from each of the six varieties were graded following the procedure outlined in the U.S. Standard for Grades of Snap Beans ${ }^{4}$. In the sensory tastings, tasters were asked to express their opinions on the following quality attributes of the produce: roughness of the skin, appearance, fibrousness, firmness, texture, color and taste. The attributes were appraised following hedonic scales of descriptive terms.

\section{RESULTS AND DISCUSSION}

Tables 1, 2, 3 and 4 show the data collected. It was observed that samples from the fall season had a content of No. 1 grade material in a range of 70 to $94 \%$ for the two picking or sampling periods (table 1). Cultivars Wade, Contender and Tendergreen showed better yields of graded beans in the second picking than in the first; Harvester, Astro and Olympia remained practically at the same level of quality for the two sampling periods. Harvester, however, had the highest content with 94.0 and $93.1 \%$ for the first and second pickings, respectively. Olympia had the lowest with 74.1 and $74.8 \%$.

Sample fractions of No. 1 grade pods were collected from the winter plots in a range of 66.9 to $93.3 \%$ (table 2). The amount of graded beans was higher in the samples collected in the first picking for Contender, Wade and Olympia than in the second picking for the same cultivars. Tendergreen, Harvester and Astro showed improvements in their yields of graded material during the second picking, 7 days after the first sampling.

\footnotetext{
${ }^{3}$ Trade names are used in this publication solely for the purpose of providing specific information. Mention of trade names does not constitute a guarantee or warranty of equipment or materials by the Agricultural Experiment Station of the University of Puerto Rico or an endorsement over other equipment or materials not mentioned.

${ }^{4}$ United States Standards for Grades of Snap Beans, USDA, Consumer and Marketing, Wash., D.C. (1936).
} 
TABLE 1.-Grading of snap beans planted during fall

\begin{tabular}{|c|c|c|c|c|c|c|c|c|c|c|c|c|}
\hline \multirow{2}{*}{$\begin{array}{c}\text { Quality } \\
\text { Attribute-\% }\end{array}$} & \multicolumn{2}{|c|}{ Wade } & \multicolumn{2}{|c|}{ Contender } & \multicolumn{2}{|c|}{ Harvester } & \multicolumn{2}{|c|}{ Astro } & \multicolumn{2}{|c|}{ Olympia } & \multicolumn{2}{|c|}{ Tendergreen } \\
\hline & $\begin{array}{c}\text { 1st. } \\
\text { picking }\end{array}$ & $\begin{array}{l}\text { 2nd. } \\
\text { picking }\end{array}$ & $\begin{array}{c}\text { Ist. } \\
\text { picking }\end{array}$ & $\begin{array}{l}\text { 2nd. } \\
\text { picking }\end{array}$ & $\begin{array}{l}\text { 1st. } \\
\text { picking }\end{array}$ & $\begin{array}{l}\text { 2nd. } \\
\text { picking }\end{array}$ & $\begin{array}{c}\text { 1st. } \\
\text { picking }\end{array}$ & $\begin{array}{l}\text { 2nd. } \\
\text { picking }\end{array}$ & $\begin{array}{c}\text { 1st. } \\
\text { picking }\end{array}$ & $\begin{array}{c}\text { 2nd. } \\
\text { picking }\end{array}$ & $\begin{array}{c}\text { 1st. } \\
\text { picking }\end{array}$ & $\begin{array}{l}\text { 2nd. } \\
\text { picking }\end{array}$ \\
\hline Decay & 0 & 0 & 0 & 0 & 0 & 0 & 0 & 0 & 0 & 0 & 0 & 0 \\
\hline Over mature & 3.0 & 6.0 & 9.1 & 4.3 & 0 & 1.9 & 3.0 & 1.9 & 7.9 & 10.0 & 3.6 & 3.6 \\
\hline Scars & 8.4 & 1.9 & 2.5 & 5.4 & 0.5 & 1.6 & 4.5 & 3.0 & 5.2 & 2.7 & 3.6 & 1.0 \\
\hline Mishapen & 5.7 & 2.5 & 7.5 & 3.9 & 2.9 & 1.9 & 3.6 & 3.9 & 7.5 & 12.5 & 5.0 & 1.4 \\
\hline Poorly developed & 2.5 & 1.4 & 7.3 & 3.4 & 2.0 & 1.0 & 3.6 & 3.6 & 3.4 & 8.0 & 3.6 & 2.7 \\
\hline Insect damage & 4.5 & 0.2 & 3.6 & 2.7 & 0.7 & 0.5 & 2.5 & 2.1 & 1.9 & 1.0 & 0 & 0 \\
\hline Cut or broken & 0 & 0 & 0 & 0 & 0 & 0 & 0 & 0 & 0 & 0 & 0 & 0 \\
\hline Total defects & 24.1 & 12.0 & 30.0 & 19.7 & 6.1 & 6.9 & 17.2 & 14.5 & 25.9 & 34.2 & 15.8 & 8.7 \\
\hline$\%$ U.S. No. I & 75.9 & 88.0 & 70.0 & 80.3 & 93.9 & 93.1 & 82.8 & 85.5 & 74.1 & 65.8 & 84.2 & 91.3 \\
\hline
\end{tabular}

TABLE 2.-Grading of snap beans planted during winter

\begin{tabular}{|c|c|c|c|c|c|c|c|c|c|c|c|c|}
\hline \multirow{2}{*}{$\begin{array}{c}\text { Quality } \\
\text { Attribute-\% }\end{array}$} & \multicolumn{2}{|c|}{ Wade } & \multicolumn{2}{|c|}{ Contender } & \multicolumn{2}{|c|}{ Harvester } & \multicolumn{2}{|c|}{ Astro } & \multicolumn{2}{|c|}{ Olympia } & \multicolumn{2}{|c|}{ Tendergreen } \\
\hline & $\begin{array}{l}\text { 1st. } \\
\text { picking }\end{array}$ & $\begin{array}{l}2 \mathrm{nd} . \\
\text { picking }\end{array}$ & $\begin{array}{l}\text { 1st. } \\
\text { picking }\end{array}$ & $\begin{array}{l}\text { 2nd. } \\
\text { picking }\end{array}$ & $\begin{array}{c}\text { lst. } \\
\text { picking }\end{array}$ & $\begin{array}{l}\text { 2nd. } \\
\text { picking }\end{array}$ & $\begin{array}{l}\text { 1st. } \\
\text { picking }\end{array}$ & $\begin{array}{l}\text { 2nd. } \\
\text { picking }\end{array}$ & $\begin{array}{c}\text { 1st. } \\
\text { picking }\end{array}$ & $\begin{array}{l}\text { 2nd. } \\
\text { picking }\end{array}$ & $\begin{array}{l}\text { 1st. } \\
\text { picking }\end{array}$ & $\begin{array}{l}\text { 2nd. } \\
\text { picking }\end{array}$ \\
\hline Decay & 0 & 0 & 0 & 0 & 0 & 0 & 0 & 0 & 0 & 0 & 0 & 0 \\
\hline Over mature & 2.0 & 11.5 & 1.4 & 0 & 0 & 0 & 0 & 0 & 0 & 2.5 & 0.9 & 0 \\
\hline Scars & 0.0 & 9.5 & 0.9 & 3.0 & 0 & 2.8 & 1.7 & 6.0 & 2.5 & 4.64 & 2.5 & 2.0 \\
\hline Mishapen & 0.9 & 4.0 & 2.7 & 3.5 & 0 & 0 & 0.5 & 0 & 5.0 & 1.07 & 6.8 & 2.14 \\
\hline Poorly developed & 9.3 & 0 & 1.7 & 4.0 & 15.18 & 1.2 & 12.9 & 2.5 & 4.8 & 2.68 & 22.9 & 3.7 \\
\hline Insect damage & 0 & 4.0 & 0 & 13.0 & 0 & 0 & 0 & 6.0 & 0 & 1.07 & 0 & 1.6 \\
\hline Cut or broken & 0 & 0 & 0 & 0 & 0 & 0 & 0 & 0 & 0 & 0 & 0 & 0 \\
\hline Total defects & 12.2 & 29.0 & 6.7 & 23.5 & 15.18 & 4.0 & 15.1 & 14.5 & 12.3 & 11.96 & 33.1 & 9.44 \\
\hline$\%$ U.S. No. 1 & 87.8 & 71.0 & 93.3 & 76.5 & 84.82 & 96.0 & 84.9 & 85.5 & 87.7 & 88.04 & 66.9 & 90.56 \\
\hline
\end{tabular}


TABLE 3.-Grading of snap beans planted during spring

\begin{tabular}{|c|c|c|c|c|c|c|c|c|c|c|c|c|}
\hline \multirow{2}{*}{$\begin{array}{c}\text { Quality } \\
\text { Attribute-\% }\end{array}$} & \multicolumn{2}{|c|}{ Wade } & \multicolumn{2}{|c|}{ Contender } & \multicolumn{2}{|c|}{ Harvester } & \multicolumn{2}{|c|}{ Astro } & \multicolumn{2}{|c|}{ Olympia } & \multicolumn{2}{|c|}{ Tendergreen } \\
\hline & $\begin{array}{c}\text { 1st. } \\
\text { picking }\end{array}$ & $\begin{array}{c}\text { 2nd. } \\
\text { picking }\end{array}$ & $\begin{array}{c}\text { 1st. } \\
\text { picking }\end{array}$ & $\begin{array}{l}\text { 2nd. } \\
\text { picking }\end{array}$ & $\begin{array}{l}\text { 1st. } \\
\text { picking }\end{array}$ & $\begin{array}{l}\text { 2nd. } \\
\text { picking }\end{array}$ & $\begin{array}{l}\text { 1st. } \\
\text { picking }\end{array}$ & $\begin{array}{c}\text { 2nd. } \\
\text { picking }\end{array}$ & $\begin{array}{c}\text { 1st. } \\
\text { picking }\end{array}$ & $\begin{array}{c}2 \text { 2nd. } \\
\text { picking }\end{array}$ & $\begin{array}{c}\text { 1st. } \\
\text { picking }\end{array}$ & $\begin{array}{c}\text { 2nd. } \\
\text { picking }\end{array}$ \\
\hline Decay & 0 & 0 & 0 & 0 & 0 & 0 & 0 & 0 & 0 & 0 & 0 & 0 \\
\hline Over mature & 2.86 & 3.57 & 1 & 1 & 2.14 & 0 & 2.14 & 0 & 5.35 & 8.93 & 2.14 & 5.18 \\
\hline Scars & 2.5 & 7.31 & 6 & $\Leftrightarrow$ & 0 & 4.82 & 2.68 & 5.9 & 4.82 & 4.29 & 7.5 & 3.57 \\
\hline Mishapen & 5.0 & 7.32 & 2 & $\stackrel{\mathbb{2}}{\stackrel{\sim}{\Omega}}$ & 1.79 & 2.5 & 1.25 & 0.89 & 1.96 & 8.2 & 3.04 & 2.86 \\
\hline Poorly developed & 3.04 & 5.71 & 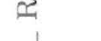 & జ & 1.42 & 0.89 & 2.14 & 1.43 & 2.14 & 2.68 & 2.32 & 1.96 \\
\hline Insect damage & 0 & 1.43 & $i$ & 1 & 0 & 0.89 & 0 & 0.89 & 1.78 & 1.43 & 0 & 1.07 \\
\hline Total defects & 13.4 & 25.34 & & & 5.35 & 9.1 & 8.21 & 9.11 & 16.05 & 25.53 & 15.0 & 14.64 \\
\hline$\%$ U.S. No. 1 & 86.6 & 74.66 & & & 94.65 & 90.9 & 91.79 & 90.89 & 83.95 & 74.47 & 85.0 & 85.36 \\
\hline
\end{tabular}

TABLE 4.-Grading of snap beans planted during summer

\begin{tabular}{|c|c|c|c|c|c|c|c|c|c|c|c|c|}
\hline \multirow{2}{*}{$\begin{array}{c}\text { Quality } \\
\text { Attribute \% }\end{array}$} & \multicolumn{2}{|c|}{ Wade } & \multicolumn{2}{|c|}{ Contender } & \multicolumn{2}{|c|}{ Harvester } & \multicolumn{2}{|c|}{ Astro } & \multicolumn{2}{|c|}{ Olympia } & \multicolumn{2}{|c|}{ Tendergreen } \\
\hline & $\begin{array}{c}\text { 1st. } \\
\text { picking }\end{array}$ & $\begin{array}{l}\text { 2nd. } \\
\text { picking }\end{array}$ & $\begin{array}{l}\text { 1st. } \\
\text { picking }\end{array}$ & $\begin{array}{l}\text { 2nd. } \\
\text { picking }\end{array}$ & $\begin{array}{l}1 \text { st. } \\
\text { picking }\end{array}$ & $\begin{array}{c}2 \mathrm{nd} . \\
\text { picking }\end{array}$ & $\begin{array}{l}\text { Ist. } \\
\text { picking }\end{array}$ & $\begin{array}{c}2 \mathrm{nd} . \\
\text { picking }\end{array}$ & $\begin{array}{l}\text { 1st. } \\
\text { picking }\end{array}$ & $\begin{array}{l}\text { 2nd. } \\
\text { picking }\end{array}$ & $\begin{array}{l}\text { lst. } \\
\text { picking }\end{array}$ & $\begin{array}{c}\text { 2nd. } \\
\text { picking }\end{array}$ \\
\hline Decay & 1.62 & 4.00 & 1.08 & 19.16 & 8.24 & 6.38 & 0 & 4.66 & 0 & 9.19 & 0 & 6.65 \\
\hline Over mature & 1.27 & 1.49 & 0 & 5.76 & 0 & 5.11 & 0 & 0 & 1.73 & 0 & 0 & 17.32 \\
\hline Scars & 15.46 & 19.07 & 5.04 & 30.53 & 7.41 & 8.52 & 24.42 & 14.13 & 6.15 & 21.18 & 4.93 & 21.07 \\
\hline Mishapen & 2.13 & 9.37 & 6.17 & 13.97 & 17.66 & 7.55 & 5.65 & 1.38 & 23.36 & 17.97 & 1.69 & 3.91 \\
\hline Poorly developed & 0.54 & 0.37 & 1.48 & 0.19 & 1.37 & 2.92 & 0.23 & 3.83 & 0 & 0 & 0.11 & 0.87 \\
\hline Insect damage & 6.48 & 8.39 & 6.07 & 12.36 & 5.40 & 16.94 & 3.95 & 9.41 & 6.12 & 10.47 & 7.34 & 6.24 \\
\hline Cut or broken & 0.65 & 1.08 & 4.05 & 2.33 & 4.90 & 8.81 & 2.03 & 2.68 & 0.47 & 3.85 & 1.54 & 0 \\
\hline Total defects & 28.15 & 43.77 & 23.89 & 84.30 & 44.98 & 56.23 & 36.28 & 36.09 & 37.83 & 62.66 & 15.61 & 56.06 \\
\hline$\%$ U.S. No. 1 & 71.85 & 56.23 & 76.11 & 15.70 & 55.02 & 43.77 & 63.72 & 63.91 & 62.17 & 37.34 & 84.39 & 43.44 \\
\hline
\end{tabular}


The range of graded pods for the two sampling periods of the spring plots was from 74.5 to $94.7 \%$ (table 3). The data shows higher content of No. 1 grade beans in the first picking of Harvester, Astro, Wade and Olympia. Tendergreen, with $85.0 \%$ of No. 1 grade pods, remained at the same level for the two pickings. On the other hand, no information was obtained from the spring planting of Contender because of rust damage before sampling.

The summer plantings showed the following ranges in their yields of graded beans: 55.0 to $84.4 \%$ in the first picking and 15.7 to $63.9 \%$ in the second sampling. These were the lowest yields of graded material obtained for these varieties in any of the four planting seasons of the study.

During the course of the study, it was observed that the yield of high quality grade beans depended on the planting season and on the age of the pods at the time of harvesting. Some varieties showed their highest yield of graded material at 45 or 47 days after planting, whereas others

TABLE 5.-Arrangement of the six bean varieties according to their yields of US No. 1 grade pods for each particular planting season

\begin{tabular}{|c|c|c|c|}
\hline Season & $\begin{array}{c}\text { One picking } \\
\text { At } 45 \text { or } 47 \text { days after } \\
\text { planting }\end{array}$ & $\begin{array}{c}\text { One picking } \\
\text { At } 52 \text { or } 54 \text { days after } \\
\text { planting }\end{array}$ & $\begin{array}{l}\text { Two pickings } \\
\text { combined } \\
\text { At } 45 \text { and } 52 \text { days } \\
\text { after planting }\end{array}$ \\
\hline Fall & $\mathrm{H} A \mathrm{~T} \quad \mathrm{~W} O \mathrm{C}^{1}$ & $\mathrm{H} \mathrm{T}$ W A $\mathrm{C} O$ & 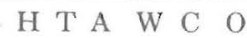 \\
\hline Winter & 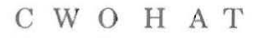 & $\mathrm{T} \mathrm{H} \quad \mathrm{A} \quad \mathrm{O} \quad \mathrm{C}$ W & $\mathrm{H} \mathrm{A} \mathrm{C} \mathrm{O} \quad \mathrm{W}$ T \\
\hline Spring & $\mathrm{HA}$ A $\mathrm{T} O \mathrm{O}$ & $\mathrm{HA} \mathrm{T}$ W $\mathrm{OC}$ & 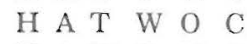 \\
\hline Summer & $\mathrm{T} \mathrm{C}$ W $\mathrm{A} \mathrm{OH}$ & A W $\mathrm{T}$ H O C & T A W O H C \\
\hline
\end{tabular}

' A, Astro; C, Contender; H, Harvester; O, Olympia; T, Tendergreen; W, Wade.

exhibited their peaks about 7 days later, that is, at 52 or 54 days after setting the plots.

In an effort to establish a comparison among the varieties, an arrangement is presented in table 5 , in which the cultivars are arranged according to their yield of No. 1 grade material in each planting season and for each of the two harvesting schemes used for the collection of the samples. In the table the varieties are represented by symbols that are arranged from left to right in the order of the higher to lower yielders of graded beans in each particular planting season. A third column has been included to present the varieties arranged according to the average yields obtained by combining both the first and second pickings.

The results of the sensory evaluation showed no significant difference in quality. Planting season seemed to have no influence on the taste of the beans.

\section{RESUMEN}

En la Subestación de Isabela se llevó a cabo un experimento con habichuelas tiernas de las variedades Astro, Contender, Harvester, Olympia, Tendergreen y Wade para determinar 
el efecto de la época de siembra y el de la edad de la planta al momento de la cosecha sobre la calidad del fruto.

Las seis variedades se sembraron en las diferentes épocas del año. Se hizo la primera cosecha a los 45-47 días de haberse sembrado y la segunda 7 días después. Se determinó la contidad de habichuelas de marca número 1 en cada una de las muestras cosechadas para comparar las variedades con relación a la calidad de la habichuela producida.

Las muestras de habichuelas cosechadas en otoño, invierno y primavera fueron de mejor calidad que las que se cosecharon en el verano. Las variedades Astro y Tendergreen fueron las de mejor rendimientos de marca No. 1 entre las cosechadas en el verano aunque tan solo produjeron 63.8 y $63.9 \%$, respectivamente. En las siembras de otoño y primavera las muestras de la variedad Harvester cosechadas a los 45 y los 52 dias de sembradas, produjeron los mejores rendimientos de marca No, 1 entre las seis variedades estudiadas. 